\title{
Cost Analysis of an Automated and Manual Cataloging and Book Processing System
}

Joselyn DRUSCHEL: Washington State University, Pullman.

A comparative cost analysis of an automated network system (WLN) and a local manual system of cataloging and book processing at Washington State University Libraries indicates that the automated system is about 20 percent less costly than the manual system. A per-unit cost approach was used in calculating the monthly cost of each system based on the average number of items processed per month under the automated system. The process and the results of the analysis are presented in a series of charts which detail the tasks, items processed, unit and total monthly costs of both the manual and automated systems. The higher costs of the manual system were essentially staff costs.

The Technical Services Division (TSD) of Washington State University Libraries (WSUL) has had considerable experience in the use of automated techniques in selected areas of technical processing. An in-house automated acquisitions system was developed and implemented in 1967; that in-house system was eventually replaced by the acquisitions component of the Washington Library Network (WLN). Since November 1977, the Technical Services Division of WSUL has used the WLN bibliographic component for data verification (searching) and cataloging of materials.

Although the Library has generally known its total automation expenditures, it has lacked a more precise breakdown of cost data on automated processing. Moreover, the library has practically no cost data on manual processing. This report deals only with the costs of using the WLN bibliographic system, not the WLN acquisitions component. An analysis was made of the total costs of both the automated and manual book processing systems. The objectives in undertaking the cost analysis were threefold: (1) to identify the essentially unknown costs of manual processing; (2) to provide more exact cost data on automated processing; and (3) to develop comparable data on the costs of each system.

Manuscript received October 1980; accepted December 1980. 


\section{METHODOLOGY}

The methodology used in this cost analysis was a per-unit cost approach. First, each process or task in which the staff were engaged in cataloging and book processing was identified. Second, the per-unit cost-e.g., staff, data base, materials - of each process was calculated. Finally, monthly costs were determined by multiplying the average number of items processed per month by the unit cost per task. The cost analysis charts (tables $1(\mathrm{a})-1(\mathrm{e})$ - manual system; tables 2(a)-2(d) - automated system), which detail the tasks, items processed, and unit and total costs form the body of the analysis. Equipment costs-purchase, lease, maintenance-were calculated separately, and are included in the summary cost data for each system (table 3 ).

\section{IDENTIFICATION OF PROCESSES}

The staff of the TSD Cataloging and Book Processing Unit perform the following functions: bibliographic verification, bibliographic record production, bibliographic record maintenance, the marking of materials, binding preparation and receipt (for most of the library system), and the preparation of book cards.

Table 1(a). Cost Analysis: Manual Cataloging and Book Processing System

\begin{tabular}{|c|c|c|c|c|c|c|c|}
\hline Process & $\begin{array}{l}\text { Staff } \\
\text { Costs } \\
\text { Per } \\
\text { Item }\end{array}$ & $\begin{array}{c}\text { Data } \\
\text { Base } \\
\text { Costs/ } \\
\text { Item }\end{array}$ & $\begin{array}{l}\text { Subscription } \\
\text { Costs/Item }\end{array}$ & $\begin{array}{l}\text { Materials } \\
\text { Costs/Item }\end{array}$ & $\begin{array}{l}\text { Total } \\
\text { Cost } \\
\text { Per } \\
\text { Item }\end{array}$ & $\begin{array}{c}\text { Average } \\
\text { Number } \\
\text { Processed } \\
\text { Per } \\
\text { Month }\end{array}$ & $\begin{array}{c}\text { Total } \\
\text { Cost } \\
\text { Per } \\
\text { Month }\end{array}$ \\
\hline $\begin{array}{l}\text { Bibliographic Searching } \\
\text { IDC Microfiche Search (LC and } \\
\text { CIP Copy) }\end{array}$ & & & & & & & \\
\hline LT I (.084/min@3 min/item) & \$. 252 & & $\$ .21$ & · & $\$ .462$ & 2484 & $\$ 1,148$ \\
\hline LT II (.094/min@3 min/item) & .282 & & .21 & & .492 & 496 & 244 \\
\hline LT III (.117/min@3min/item) & .351 & & .21 & & .561 & 992 & 557 \\
\hline \multicolumn{8}{|l|}{$\begin{array}{l}\text { Subscription costs-IDC } \\
(\$ 10,000 / \mathrm{yr} \div 47,664 \\
\text { searches } / \mathrm{yr}=.21 / \text { search })\end{array}$} \\
\hline Microfiche Search Subtotal & & & & & & 3972 & $\$ 1,949$ \\
\hline \multicolumn{8}{|l|}{$\begin{array}{l}\text { National Union Catalog, etc., } \\
\text { Search }\end{array}$} \\
\hline LT I (.084/min@15 min/item) & $\$ 1.26$ & & $\$ .19$ & & $\$ 1.45$ & 588 & \& 853 \\
\hline LT II (.094/min@15 min/item) & 1.41 & & .19 & & 1.60 & 169 & 270 \\
\hline $\begin{array}{l}\text { LT III (.117/min@ @20 } \\
\text { min/item) }\end{array}$ & 2.34 & & .19 & & 2.53 & 418 & 1,058 \\
\hline $\begin{array}{l}\text { LT III (.117/min @ } 40 \\
\text { min/item) }\end{array}$ & 4.68 & & .19 & & 4.87 & 100 & 487 \\
\hline \multicolumn{8}{|l|}{$\begin{array}{l}\text { Subscriptions }(\$ 2,940 / \mathrm{yr} \div \\
15,300 \text { searches } / \mathrm{yr}= \\
.19 / \text { search })\end{array}$} \\
\hline Manual Search Subtotal & & & & & & 1275 & $\$ 2,668$ \\
\hline Bibliographic Searching Total & & & & & & 5247 & $\$ 4,617$ \\
\hline
\end{tabular}


Table 1(b). Cost Analysis: Manual Cataloging and Book Processing System

\begin{tabular}{|c|c|c|c|c|c|c|c|}
\hline Process & $\begin{array}{l}\text { Staff } \\
\text { Costs } \\
\text { Per } \\
\text { Item }\end{array}$ & $\begin{array}{c}\text { Data } \\
\text { Base } \\
\text { Costs/ } \\
\text { Item }\end{array}$ & $\begin{array}{c}\text { Subscription } \\
\text { Costs/Item }\end{array}$ & $\begin{array}{l}\text { Materials } \\
\text { Costs/Item }\end{array}$ & $\begin{array}{c}\text { Total } \\
\text { Cost } \\
\text { Per } \\
\text { Item }\end{array}$ & $\begin{array}{c}\text { Average } \\
\text { Number } \\
\text { Processed } \\
\text { Per } \\
\text { Month }\end{array}$ & $\begin{array}{c}\text { Total } \\
\text { Cost } \\
\text { Per } \\
\text { Month }\end{array}$ \\
\hline
\end{tabular}

Bibliographic Record

Production-Processing and

Products

1. Cataloging with LC Microfiche

Copy

Type abbreviated fanfold

(4-part $3 \times 5$ slips)

Timeslip (.03/min@min/item $x$ 72)

LT I (.084/min@10 min/item fanfold $x$ 985)

Check Series

LT I (.084/min@2min/item)

Revise fanfold

Supervisor I (.126/min @ 3 $\mathrm{min} / \mathrm{item}$ )

Check fanfold against book; separate fanfold

LT II (.094/min @ 2 min/item x 540)

Supervisor I (.126/min@2 $\mathrm{min} /$ item $\times 517$ )

Arrange and file shelflist copy of fanfold

Timeslip (.03/min@1.5 $\mathrm{min} / \mathrm{slip}$ )

Revise shelflist filing of fanfold slips

LT II (.094/min@ @ min/slip)

Verify Authorities (Subject and name) (1057x4)

Timeslip (.03/min@4 $\mathrm{min} /$ item)

Type multilith master for card production

LT I (.084/min @6min/master)

$.06 /$

Revise typed multilith master

LT I (.084/min@3 min/master)

Run multilith masters

Multilith Operator (.13/min@ $3.5 \mathrm{~min} / \mathrm{set}$ )

Microfiche Copy Cataloging Subtotal

master

2. Cataloging with Modified Copy (NUC/LC)

Type fanfolds (4-part $3 \times 5$ slips)

LT I (.084/min@ @15 min/item) \$1.26 
Table 1(b) (cont.)

Check Series

LT I (.084/min@2 min/item) .168

Revise fanfold

Supervisor I (.126/min @ 5

$\mathrm{min} /$ item)

Review fanfold cataloging

Librarian(.155/min@5

$\mathrm{min} / \mathrm{item})$

Separate fanfolds

LT II (.094/min@30 sec/item)

Arrange and file shelflist copy of fanfold

Timeslip (.03/min@1.5 $\mathrm{min} / \mathrm{slip}$ )

Revise filing of shelflist copy

LT II (.094/min @ 1 min/slip)

Verify authorities $(984 \times 4)$

Timeslip (.03/min@4 $\mathrm{min} /$ item)

Type multilith master for card production

LT I (.084/min@6min/master)

$.06 /$

master

Revise typed multilith master

LT I (.084/min@3 min/master)

Run multilith masters

Multilith Operator(.13/min@ $3.5 \mathrm{~min} / \mathrm{set}$ )

\section{Modified Copy Cataloging} Subtotal

Librarian(.205/min@60 $\mathrm{min} /$ item $\times 22$ )

Revise cataloging

Librarian(.205/min@5

$$
\mathrm{min} / \text { item) }
$$

Type fanfolds (4-part $3 \times 5$ slips)

LT I (.084/min@15 min/item)

\section{Check Series}

LT I (.084/min@2 min/item

Revise fanfold

Supervisor I (.126/min@5 $\mathrm{min} / \mathrm{item}$ )

\begin{tabular}{c} 
(Cost of \\
cards \\
see below) \\
\hline
\end{tabular}

\section{$\$ .08 /$}

$\$ 4.43$

984

$\$ 4,359$

Separate fanfolds

LT II (.094/min@30 sec/item)

Arrange and file shelflist copy of fanfold

Timeslip (.03/min@1.5 $\mathrm{min} /$ item)

Revise filing of shelflist copy

LT II (.094/min @ 1 min/slip) 
Table 1(b) (cont.)

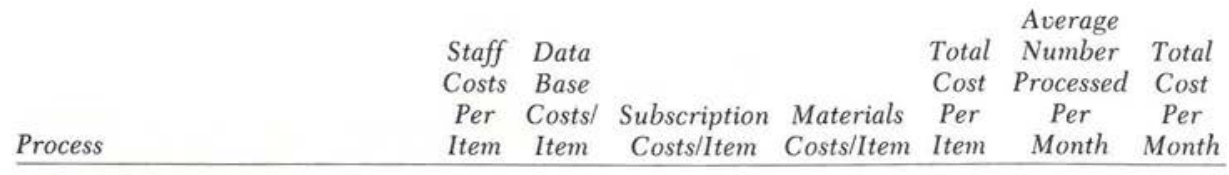

Type multilith master for card production

LT I (.084/min@6min/master) .504

$.06 /$

master

Revise typed master

LT I (.084/min@3 min/master)

Run multilith master

Multilith Operator (.13/min @

$3.5 \mathrm{~min} / \mathrm{set}$ )

Original Cataloging Subtotal $\overline{\$ 14.085}$

Catalog Cards $(7$ cards/set @ $.055 /$ card)

Subtotal

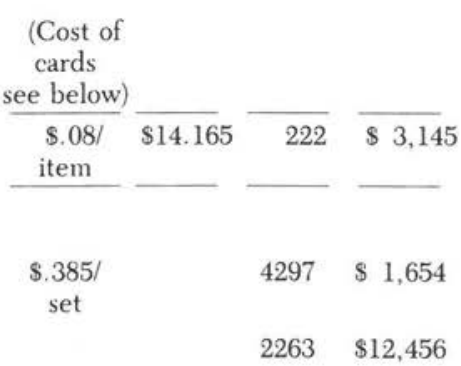

Cataloging Total

$2263 \$ 12,456$

4. Miscellaneous Bibliographic

Record Production

Assign class numbers to theses

Supervisor I (.126/min@2 $\mathrm{min} / \mathrm{item})$

Assign subject headings for audio visual materials

Librarian (.155/min @ 2 $\mathrm{min} / \mathrm{set}$ )

Type multilith masters for catalog cards for A-V materials

LT I (.084/min @ 6 min/master) .504

Revise multilith masters

LT I (.084/min@3 min/master)

Multilith Operator (.13/min @ $3.5 \mathrm{~min} / \mathrm{set}$ ) (20 cards)

Resolve problems; general supervision

Supervisor I (7.56/hr x 52 hrs/mo)

Librarian (9.32/hr@22 hrs/mo)

Miscellaneous Bibliographic Record Production Subtotal

Bibliographic Record

Production Total 
Table 1(c). Cost Analysis: Manual Cataloging and Book Processing System

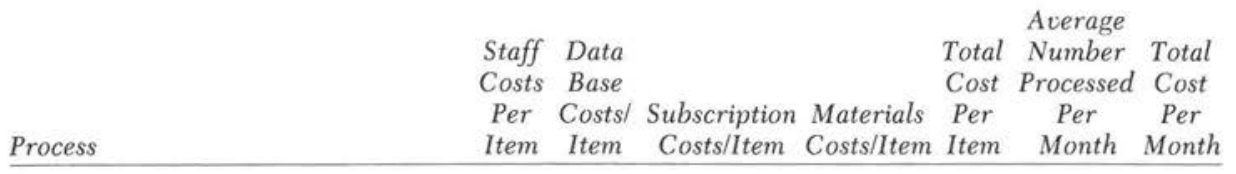

Bibliographic Record Maintenance

Count sets of cards and match against cataloging copy

LT I (.084/min@2 sets/min)

Type subject and added entries on card sets

Timeslip (.03/min @3 min/set)

Revise card sets

LTII (.094/min@3 min/set)

LT III (.117/min@3 min/set)

Type subject and name authority slips

Timeslip (.03/min @ $1 \mathrm{~min} / \mathrm{slip})$

File subject and name authority slips

Timeslip (.03/min @ 1 min/slip)

Separate card sets

LT I (.084/min @2 sets/min)

File subject catalog cards (2263x2)

LT II (.094/min@1 min/card)

File A/T catalog cards (2263x3).

LT I (.084/min @ 1 min/card)

File shelflist cards (2)

Revise subject card filing

LT III (.117/min @ 1 min/card)

Revise A/T card filing

LT III (.117/min@1min/card)

Revise shelflist filing (2)

(094/min@1.min/card)

Supervisor I (.126/min@ @ $\mathrm{min} / \mathrm{card}$ )

Alphabetize and date workslips

LT I (.084/min@4 slips/min)

Pull card sets (withdrawals and card corrections

Timeslip (.03/min@10 min/set)

Revise card pulling (100 sets/month)

Supervisor I (.126/min@2 $\mathrm{min} / \mathrm{set}$ )

Correct card sets (50 sets/month)

Revise card corrections

Supervisor I (.126/min@2 $\mathrm{min} / \mathrm{set}$ )

Process added copies (Record accession \# on shelflist; record call \# in book; type slip for marking)

LT II (.094/min@15 min/item) 


\section{Journal of Library Automation Vol. 14/1 March 1981}

Table 1(c) (cont.)

\begin{tabular}{|c|c|c|c|c|c|c|c|}
\hline rocess & $\begin{array}{l}\text { Staff } \\
\text { Costs } \\
\text { Per } \\
\text { Item }\end{array}$ & $\begin{array}{c}\text { Data } \\
\text { Base } \\
\text { Costs/ } \\
\text { Item }\end{array}$ & $\begin{array}{c}\text { Subscription } \\
\text { Costs/Item }\end{array}$ & $\begin{array}{l}\text { Materials } \\
\text { Costs/Item }\end{array}$ & $\begin{array}{l}\text { Total } \\
\text { Cost } \\
\text { Per } \\
\text { Item }\end{array}$ & $\begin{array}{c}\text { Average } \\
\text { Number } \\
\text { Processed } \\
\text { Per } \\
\text { Month }\end{array}$ & $\begin{array}{c}\text { Total } \\
\text { Cost } \\
\text { Per } \\
\text { Month }\end{array}$ \\
\hline
\end{tabular}

Locate materials in process

LT II (.094/min@ @ 15 min/item) $\quad \$ 1.41 \quad \$ 1.41 \quad 50 \quad \$ 71$

Prepare books for binding decision

Supervisor I (.126/min@1 $\mathrm{min} /$ item)

General Supervision

Librarian(\$12.34/hr@65

hours/month)

Bibliographic Record

Maintenance Total

$\$ 6,402$

Table 1(d). Cost Analysis: Manual Cataloging and Book Processing System

\begin{tabular}{|c|c|c|c|c|c|c|c|}
\hline Process & $\begin{array}{c}\text { Staff } \\
\text { Costs } \\
\text { Per } \\
\text { Item }\end{array}$ & $\begin{array}{c}\text { Data } \\
\text { Base } \\
\text { Costs/ } \\
\text { Item }\end{array}$ & $\begin{array}{c}\text { Subscription } \\
\text { Costs/Item }\end{array}$ & $\begin{array}{c}\text { Materials } \\
\text { Costs/Item }\end{array}$ & $\begin{array}{c}\text { Total } \\
\text { Cost } \\
\text { Per } \\
\text { Item }\end{array}$ & $\begin{array}{c}\text { Average } \\
\text { Number } \\
\text { Processed } \\
\text { Per } \\
\text { Month }\end{array}$ & $\begin{array}{c}\text { Total } \\
\text { Cost } \\
\text { Per } \\
\text { Month }\end{array}$ \\
\hline
\end{tabular}

Marking

Sort materials for processing (marking)

OA II-Typing (.105/min@ $30 \mathrm{sec}$ item)

Place materials on table

OA II-Typing (.105/min@ 20 items $/ \mathrm{min}$ )

Process materials (type and paste labels, pockets, \& date due slips; type book cards)

Timeslip (.03/min@20 $\mathrm{min} / \mathrm{item}$ )

.60

Process materials with tab book cards (type and paste labels, pockets, \& date due slips)

Timeslip (.03/min@16 $\mathrm{min} / \mathrm{item}$ )

OA II-Typing (.105/min @ $16 \mathrm{~min} / \mathrm{item})$

$$
\begin{gathered}
\text { \$.029/ } \\
\text { label; } \\
\text { pocket; } \\
\text { date due } \\
\text { slip; } \\
\text { book card }
\end{gathered}
$$

label; $\quad 1.712 \quad 308$ date due slip; book card 
Table 1(d) (cont.)

Keypunch bookcards

LT I (.084/min@2.4 $\mathrm{min} / \mathrm{card}$ )

Verify book cards

LT III (.117/min @ 1.6 $\mathrm{min} /$ card)

Revise Processing

$\begin{array}{lll}.168 & 1500 \quad 252\end{array}$

LT III (.117/min@2 $\mathrm{min} /$ item)

Sort materials for delivery

OA II-Typing (.105/min@ 1.5 items $/ \mathrm{min}$ )

Unpack bindery materials, pull slips

LT I (.084/min@1 min/item)

Verify bindery slips; check price

LT III (.117/min@2 $\mathrm{min} / \mathrm{item}$ )

General supervision, bindery account of statistical data

LT III (7.04/hr@I5hrs/mo)

Supervisor II (8.97/hr@128 $\mathrm{hrs} / \mathrm{mo}$ )

Librarian(12.34/hr@15 $\mathrm{hrs} / \mathrm{mo}$ )

Marking Total

Cataloging and Book Processing Total

Table 1(e). Total Monthly Costs (Summary)

$\begin{array}{ccccc}\begin{array}{l}\text { Staff Costs } \\ \text { Per Month }\end{array} & \begin{array}{c}\text { Data Base } \\ \text { Costs/Month }\end{array} & \begin{array}{c}\text { Subscription } \\ \text { Costs } \\ \text { Per Month }\end{array} & \begin{array}{c}\text { Material Costs } \\ \text { Per Month }\end{array} & \begin{array}{c}\text { Total Cost } \\ \text { Per Month }\end{array} \\ \$ 25,775 & & \$ 1,076 & \$ 1,942 & \$ 28,793\end{array}$

Table 2(a). Cost Analysis: Automated Cataloging and Book Processing System

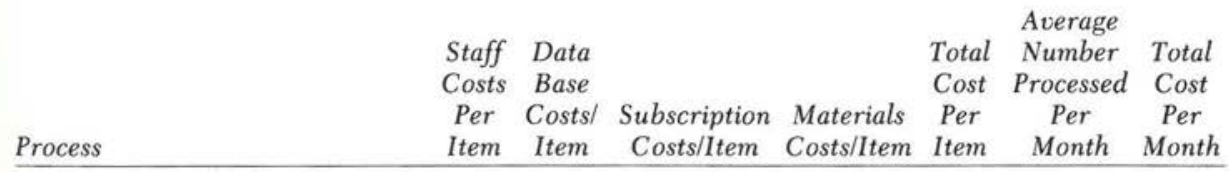

Bibliographic Searching

1. WLN Data Base Search

Items searched, no Inquiry charges

LT II (.094/min@1 min/item)

Terminal use(4@.06) 
Table 2(a) (cont.)

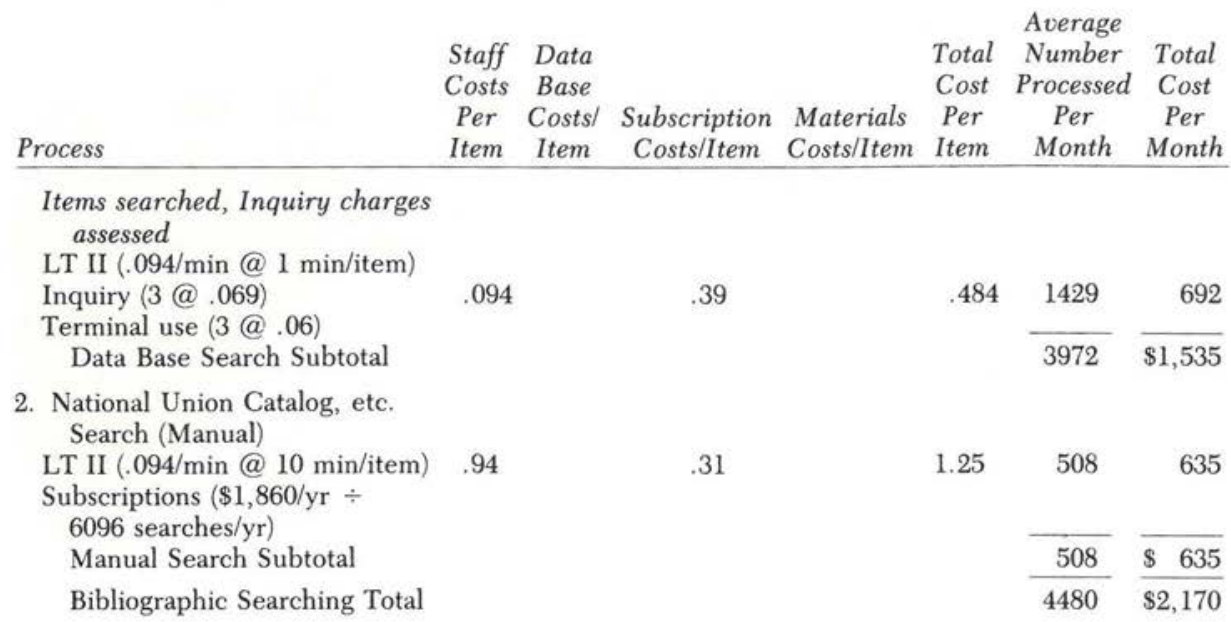

Table 2(b). Cost Analysis: Automated Cataloging and Book Processing System

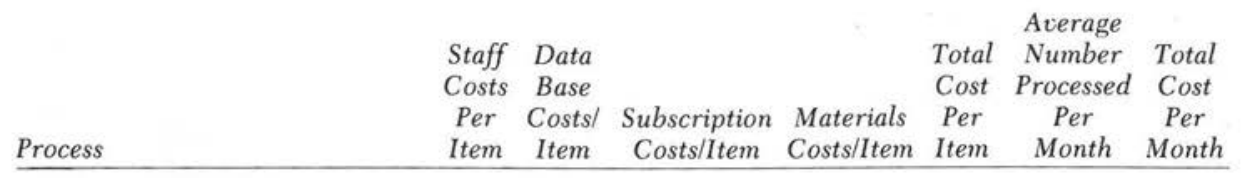

Bibliographic Record

Production-Processing and

Products

1. Materials Cataloged

Via WLN

a. Cataloging with WLN Data base copy

Attach holdings; order cards

LT II (.094/min@6 $\mathrm{min} /$ item)

Data Base Costs

Inquiry costs (no charge)

Cost per record use

Cost per request

$\$ 1.60$

Shelflist cards (4@.055)

COM (cost per record)

Terminal use (1@.06/use)

WLN Data Base Copy Subtotal

b. Cataloging with CIP Copy

Upgrade data base copy

LT II (.094/min@11 $\mathrm{min} /$ item)

Revise upgraded copy

Librarian(.155/min@5 $\mathrm{min} / \mathrm{item}$ )

Attach holdings order cards

LT II (.094/min@6 $\mathrm{min} /$ item) 
Table 2(b) (cont.)

Data Base Costs

Cost per record use $\quad \$ 1.60$

Cost per request $\quad .15$

Shelflist cards (4@.055) $\quad .22$

COM (cost per record) $\quad .43$

Terminal use (1@.06/use) ___ . .06 CIP Copy Subtotal $\overline{\$ 2.373} \overline{\$ 2.46}$

$\overline{\$ 4.833} \overline{153} \overline{\$ 739}$

c. Cataloging with Modified

Copy (e.g., NUC/LC

copy)

Prepare cataloging

worksheets

LT II (.094/min@15

$\mathrm{min} /$ item)

$\$ 1.41$

Revise cataloging

worksheets

LT II (.094/min@10

$\mathrm{min} / \mathrm{item}$ )

MARC TAG Worksheets

Supervisor II (.15/min@15

$\mathrm{min} /$ item)

Revise MARC tagged worksheets

Librarian(.155/min@8 $\mathrm{min} / \mathrm{item}$ )

Input cataloging data; attach holdings; order cards

Timeslip (.03/min@25 $\mathrm{min} / \mathrm{item}$ )

Revise data input and verify authorities

Librarian(.155/min@10 $\mathrm{min} /$ item)

Data base costs

Cost of input per record

Cost of Authority checks

(7 checks@.069/entry)

Shelflist cards (4@.055)

COM (cost per record)

Terminal use (7@.06/use) Modified Copy Subtotal

$\$ 8.14 \$ 1.69$

$\$ .14$

d. Original Cataloging

Catalog and MARC Tag material

Librarian(.155/min@60 $\mathrm{min} /$ item)

Revise Cataloging and MARC tagging

Librarian(.205/min@5 $\mathrm{min} /$ item)

Input Cataloging data; attach holdings; order cards

LT II (.094/min@ @ 25 min x 104) 


\section{Journal of Library Automation Vol. 14/1 March 1981}

Table 2(b) (cont.)

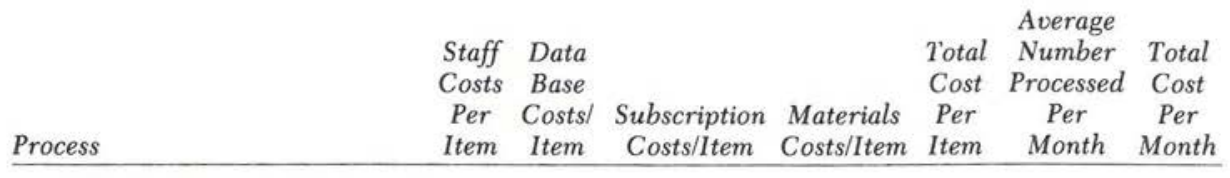

Timeslip(.03/min@ @25 min $\mathrm{x} 118$ )

Revise input; verify authorities

Librarian(.155/min@10 $\mathrm{min} / \mathrm{item})$

Data base costs

Cost of input per record

Cost of authority checks ( 7 checks@.069/entry) .48

Shelflist cards (4@.055) .22

COM (cost per record) $\quad .43$

Terminal use (7@.06/use)

Subtotal

$\overline{\$ 13.37} \$ 1.69$

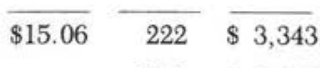

WLN Cataloging Total

2. Materials Cataloged via Other Methods

a. Microform Cataloging from Publisher's Copy

Review and revise copy; complete processing; revise card sets

Librarian(.25/min@2.7 $\mathrm{min} / \mathrm{item}$ )

Xerox card sets $(10$ cards/set)

Timeslip (.03/min@ @ 1 $\mathrm{min} /$ title)

b. Cataloging Music Scores

Catalog scores; prepare for card production; revise card sets

Librarian(.25/min@28 $\mathrm{min} / \mathrm{item})$

Xerox card sets (14 cards/set)

Timeslip (.03/min@2 $\mathrm{min} / \mathrm{title})$

Music Score Subtotal $\overline{\$ 7.06}$

Non-WLN Cataloging Total 
Table 2(b) (cont.)

3. Miscellaneous Costs

Assign class numbers to theses

Supervisor II (.15/min@2 min/item)

$\$ .30$

$\begin{array}{lll}30 & \$ & 9\end{array}$

Retrieve "RUSH" monographs

Supervisor II (.15/min@ 15 $\mathrm{min} / \mathrm{item})$

Correct/update WLN data base information

LT II (.094/min @ 10 $\mathrm{min} / \mathrm{item})$

Terminal use (1@.06/use)

Assign Subject Headings for Audio Visual Materials

Librarian(.155/min@2 $\mathrm{min} / \mathrm{set}$ )

File Subject Authority Slips for Microform Materials

Librarian(.155/min@1.15 $\mathrm{min} / \mathrm{slip}$ )

Resolve Problems; General Supervision

LT II $(5.68 / \mathrm{hr} \times 13 \mathrm{hrs} / \mathrm{mo})$

Supervisor II $(8.97 \mathrm{hrs}$ x 89 $\mathrm{hrs} / \mathrm{mo})$

Librarian $(\$ 12.34 \mathrm{hr} \times 52$ $\mathrm{hrs} / \mathrm{mo}$ )

Miscellaneous Costs Subtotal

Bibliographic Record

Production Total

Table 2(c). Cost Analysis: Automated Cataloging and Book Processing System

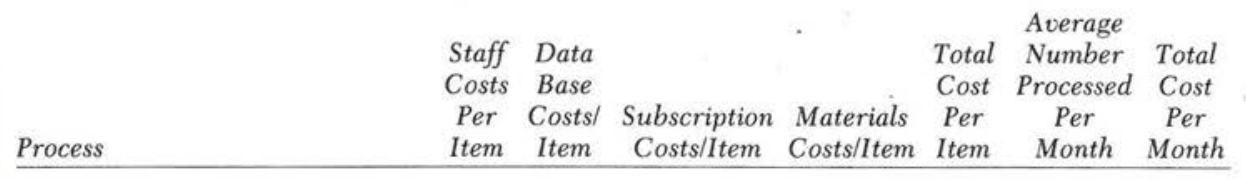

Bibliographic Record

Maintenance - TSD

Collate card sets from WLN

(7384 cards)

LT I (.083/min@ @ $30 \mathrm{sec} / \mathrm{card}) \$ .042$

$\begin{array}{llll}\$ .042 & 7384 & \$ & 310\end{array}$

Insert card sets in books

LT II (.094/min@1.6 $\mathrm{min} /$ item)

Process New Books (1846)

Review cards against books; add accession number and stamp date on shelflist card; carrect series (when needed); separate card sets and distribute

Timeslip (.03/min@10 $\mathrm{min} /$ item) 


\section{Journal of Library Automation Vol. 14/1 March 1981}

Table 2(c) (cont.)

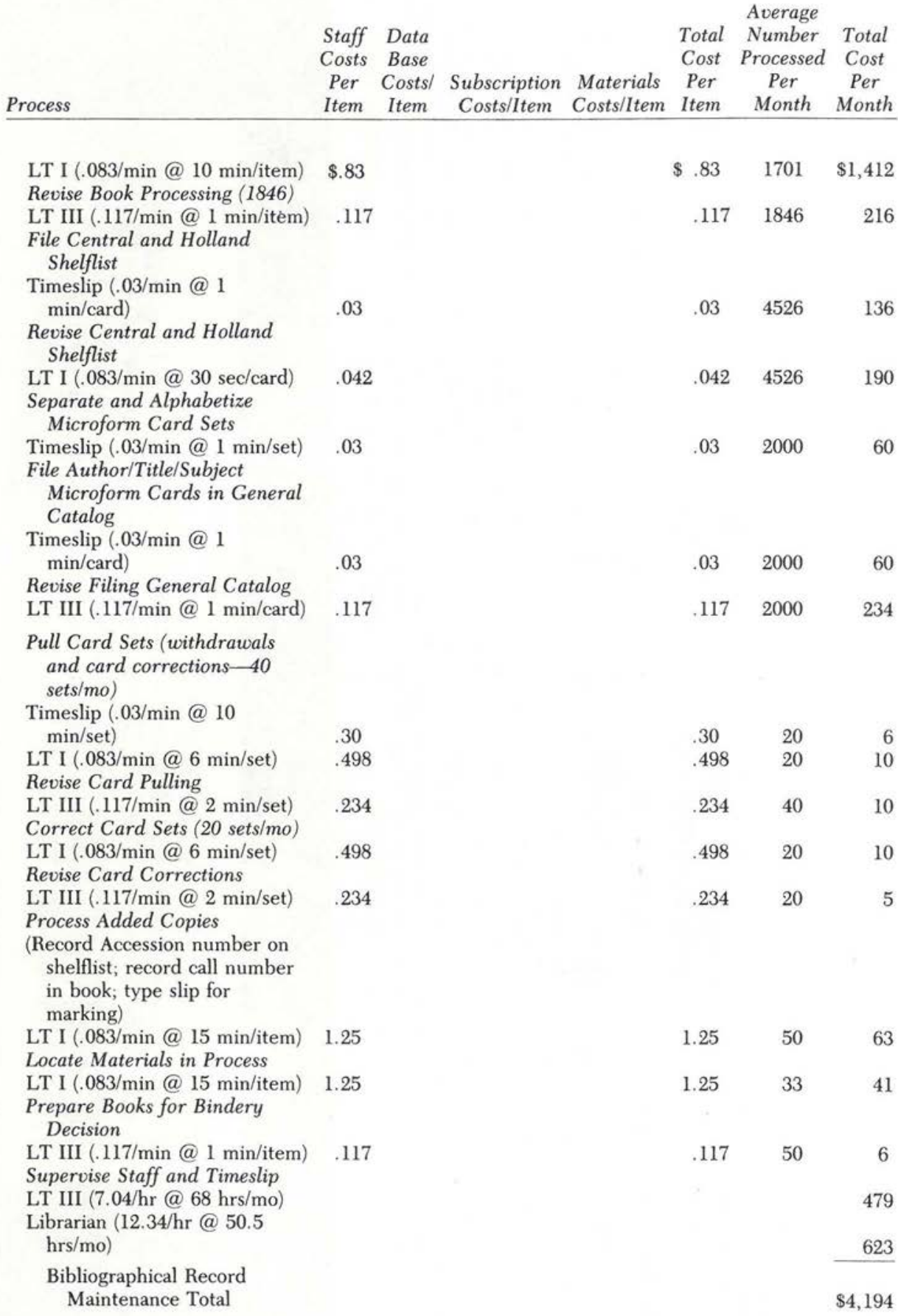


Table 2(d). Cost Analysis: Automated Cataloging and Book Processing System

\begin{tabular}{|c|c|c|c|c|c|c|c|}
\hline ocess & $\begin{array}{c}\text { Staff } \\
\text { Costs } \\
\text { Per } \\
\text { Item }\end{array}$ & $\begin{array}{c}\text { Data } \\
\text { Base } \\
\text { Costs/ } \\
\text { Item }\end{array}$ & $\begin{array}{c}\text { Subscription } \\
\text { Costs/Item }\end{array}$ & $\begin{array}{l}\text { Materials } \\
\text { Costs/Item }\end{array}$ & $\begin{array}{l}\text { Total } \\
\text { Cost } \\
\text { Per } \\
\text { Item }\end{array}$ & $\begin{array}{c}\text { Average } \\
\text { Number } \\
\text { Processed } \\
\text { Per } \\
\text { Month }\end{array}$ & $\begin{array}{l}\text { Total } \\
\text { Cost } \\
\text { Per } \\
\text { Month }\end{array}$ \\
\hline
\end{tabular}

Marking

Sort Materials for Processing (Marking)

OA II-Typing (.105/min@30 sec/item)

Place Materials on Table

OA II-Typing (.105/min@20 items/min)

Process Materials (type and paste labels, pockets and date due slips; type book cards)

Timeslip(.03/min@16 $\mathrm{min} /$ item)

Process Materials with Tab book card Book Cards (type and paste labels, pockets and date due slips)

Timeslip (.03/min@16 $\mathrm{min} / \mathrm{item}$ )

$$
.032 /
$$

OA II-Typing(.105/min@16 $\mathrm{min} / \mathrm{item}$ )

Keypunch Bookcards

LT I (.083/min@2.4 min/card) .20

date due $\quad 1.712 \quad 308 \quad 527$

slip; label; pocket; book card

Verify Book Cards

LT III (.117/min@1.6 $\mathrm{min} / \mathrm{card}$ )

Revise Processing

LT I (.083/min@2 min/item) .166

LT III (.117/min@ @ min/item).234

Sort Materials for Delivery

OA II-Typing (.105/min@ @ 1.5 items/min)

Unpack Bindery Materials; Pull slips

LT I (.083/min@ 1 item/min)

Verify Bindery Slips and Check Price

LT III (.117/min@ @ min/item)

General Supervision; Bindery

Accounts and Statistical Data

LT III (7.04/hr@36hrs/mo)

Supervisor II (8.97/hr@128 hrs/mo) 
Table 2(e). Total Monthly Costs (Summary)

$\begin{array}{ccccc}\begin{array}{l}\text { Staff Costs } \\ \text { Per Month }\end{array} & \begin{array}{c}\text { Data Base } \\ \text { Costs/Month }\end{array} & \begin{array}{c}\text { Subscription } \\ \text { Costs } \\ \text { Per Month }\end{array} & \begin{array}{c}\text { Materials Costs } \\ \text { Per Month }\end{array} & \begin{array}{c}\text { Total } \\ \text { Cost } \\ \text { Per } \text { Month }\end{array} \\ \$ 16,849 & \$ 5,480 & \$ 157 & \$ 304 & \$ 22,790\end{array}$

Table 3. Cataloging and Book Processing System: Summary Comparison Costs

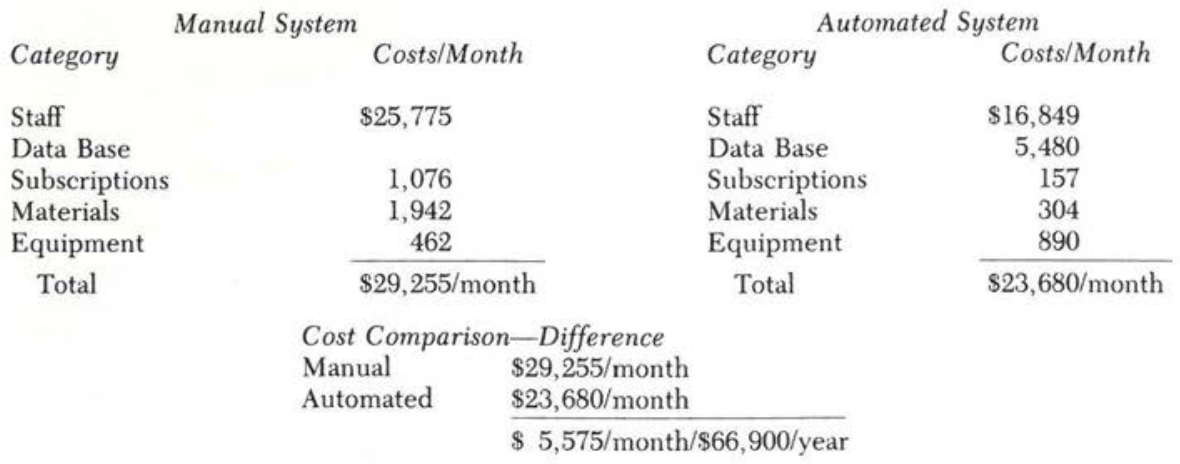

Since 1978 this unit, as well as all units in the Technical Services Division, have periodically analyzed unit activities, and recorded the data collected on Work Assignment/Staffing profile sheets (see table 4 for sample profile sheet). The primary purpose of the profiles was to develop a detailed account of work distribution throughout TSD in order to determine the staffing requirements necessary for each unit to maintain an even workflow. In the cost analysis, the Cataloging and Book Processing (CBP) profile was used to identify each unit process, as well as to provide the basic data on the number and level of staff and the time required to perform each process. Additionally, for the automated system, the CBP profile sheets, together with WLN invoices (see figure 1 for sample invoice) and WLN monthly activity reports (see figure 2 for sample activity report) were used to determine the average number of items processed per month. For example, since about 85 percent of the cataloging done in TSD is via WLN, it was possible to derive exact figures from WLN invoices for the average number of items cataloged per month. The WLN invoices also differentiated between data-base copy cataloging and original data entry. The CBP profile sheets were used to determine average number of non-WLN items cataloged.

Using a combination of WLN invoice and profile data, a chart was constructed of the average number of items searched and cataloged per month under the automated system (see table 5). In order to make costs comparable, an assumption was made that the same average number of items was searched and cataloged under the previous manual system and a similar chart was made for it (see table 6). In reality, the available staff under the manual system could not process the same amount of material per month. 
Table 4. Technical Services Division Work Assignment/Staffing Profile: November 1978

Unit: Cataloging and Book Processing. Subunit: LC Copy Editing.

\begin{tabular}{|c|c|c|c|c|c|c|}
\hline Tasks or Processes & $\begin{array}{l}\text { Average } \\
\text { Number } \\
\text { of Items } \\
\text { Received } \\
\quad \text { for } \\
\text { Processing }\end{array}$ & $\begin{array}{l}\text { Average } \\
\text { Time } \\
\text { Per Item }\end{array}$ & $\begin{array}{l}\text { Average } \\
\text { Number } \\
\text { of Items } \\
\text { Processed }\end{array}$ & $\begin{array}{c}\text { Staff } \\
\text { Hours } \\
\text { Needed } \\
\text { Per Task }\end{array}$ & $\begin{array}{c}\text { Level of } \\
\text { Staff }\end{array}$ & $\begin{array}{c}\text { Total } \\
\text { Staff } \\
\text { Hours } \\
\text { Available } \\
\text { at } \\
\text { Designated } \\
\text { Level }\end{array}$ \\
\hline \multirow{3}{*}{$\begin{array}{l}\text { Order card sets, check item } \\
\text { against data base, enter } \\
\text { holdings }\end{array}\{$} & $2100 / \mathrm{mo}$ (monos) & ) $6 \mathrm{~min} /$ item & $10 / \mathrm{hr}$ & $210 / \mathrm{mo}$ & $\{$ LT I & 124.1 \\
\hline & 2100/no(mons & & & & LT II & 85.9 \\
\hline & $63 / \mathrm{mo}$ (serials) & ) $6 \mathrm{~min} / \mathrm{item}$ & $10 / \mathrm{hr}$ & $6.3 / \mathrm{mo}$ & LT III & 6.3 \\
\hline Prepare worksheets & $210 / \mathrm{mo}$ & $10 \mathrm{~min} /$ item & $6 / \mathrm{hr}$ & $35 / \mathrm{mo}$ & $\left\{\begin{array}{l}\text { LT I } \\
\text { LT II }\end{array}\right.$ & $\begin{array}{l}17.5 \\
17.5\end{array}$ \\
\hline Prepare TSD series cards & $126 / \mathrm{mo}$ & $2 \mathrm{~min} / \mathrm{item}$ & $30 / \mathrm{hr}$ & $4.2 / \mathrm{mo}$ & LT I & 4.2 \\
\hline Do series check & $350 / \mathrm{mo}$ & $2 \mathrm{~min} / \mathrm{item}$ & $30 / \mathrm{hr}$ & $11.7 / \mathrm{mo}$ & LT I & 22.3 \\
\hline Update CIP records & $134 / \mathrm{mo}$ & $10 \mathrm{~min} / \mathrm{item}$ & $6 / \mathrm{hr}$ & $22.3 / \mathrm{mo}$ & LT II & 22.3 \\
\hline Input original cataloging data & $\{210 / \mathrm{mo}(\mathrm{mono})$ & $25 \mathrm{~min} /$ item & $2.4 / \mathrm{hr}$ & $87.5 / \mathrm{mo}$ & LT II & 87.5 \\
\hline " & 21/mo(serials) & ) $25 \mathrm{~min} / \mathrm{item}$ & $2.4 / \mathrm{hr}$ & $8.75 / \mathrm{mo}$ & LT III & 8.75 \\
\hline Process "RUSH" monographs & $168 / \mathrm{mo}$ & $15 \mathrm{~min} / \mathrm{item}$ & $4 / \mathrm{hr}$ & $42 / \mathrm{mo}$ & LT II & 42 \\
\hline $\begin{array}{l}\text { Process corrections- } \\
\text { data base information } \\
\text { Receive materials- }\end{array}$ & $360 / \mathrm{mo}$ & $10 \mathrm{~min} / \mathrm{item}$ & $6 / \mathrm{hr}$ & $60 / \mathrm{mo}$ & $\left\{\begin{array}{l}\text { LT II } \\
\text { Sup II }\end{array}\right.$ & $\begin{array}{l}30 \\
30\end{array}$ \\
\hline sort series & $2100 / \mathrm{mo}$ & 3 items $/ \mathrm{min}$ & $180 / \mathrm{hr}$ & $11.6 / \mathrm{mo}$ & LT II & 11.6 \\
\hline $\begin{array}{l}\text { Resolve problems; } \\
\text { locate materials }\end{array}$ & NA & NA & NA & $31.2 / \mathrm{m}$ & $\left\{\begin{array}{l}\text { LT II } \\
\text { LT III }\end{array}\right.$ & $\begin{array}{l}18.6 \\
13\end{array}$ \\
\hline $\begin{array}{l}\text { Prepare and sort series } \\
\text { decisions materials }\end{array}$ & $168 / \mathrm{mo}$ & $8 \mathrm{~min} /$ item & $7.5 / \mathrm{hr}$ & $22.4 / \mathrm{mo}$ & LT III & 22.4 \\
\hline Sort Mail & NA & NA & NA & $42 / \mathrm{mo}$ & LT III & 42 \\
\hline
\end{tabular}

\section{STAFF COSTS}

In the cost analysis of the automated system, the monthly wages for staff members of the Cataloging and Book Processing Unit were based on current monthly salaries (as of February 1980) plus estimated fringe benefits (21 percent). The total wages were added together for each level of staff and divided by the number of staff at that level to give an

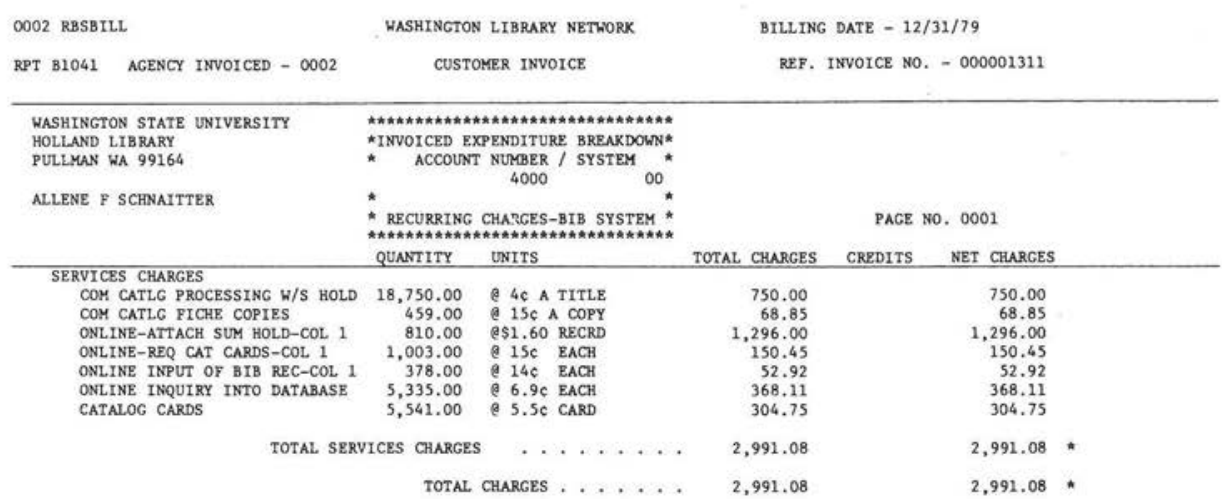

Fig. 1. Washington Library Network Customer Invoice. 
MONTHLY ACTIVITY REPORT FOR PERIOD $11 / 01 / 79$ TO $11 / 30 / 79$

\begin{tabular}{|c|c|c|c|c|c|c|c|c|}
\hline 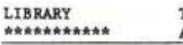 & $\begin{array}{l}\text { TOTAL } \\
\text { AS OF }\end{array}$ & $\begin{array}{l}\text { HOLDINGS } \\
11 / 30 / 79\end{array}$ & $\begin{array}{l}\text { HOLDINGS } \\
\text { ADDED }\end{array}$ & $\begin{array}{l}\text { RECORDS } \\
\text { INPUT }\end{array}$ & $\begin{array}{l}\text { CONTRIBUTION } \\
\text { FACTOR }\end{array}$ & $\begin{array}{l}\text { RCPS FROM } \\
11 / 01 \text { TO } 11 / 28\end{array}$ & $\begin{array}{l}\text { ACQ ORDERS } \\
\text { CREATED }\end{array}$ & $\begin{array}{l}\text { INQUIRY } \\
\text { TRANSACTIONS }\end{array}$ \\
\hline WaPaC & & 2,059 & 38 & 0 & $.0 z$ & 0 & 0 & 311 \\
\hline WaP1P & & 41,549 & 416 & 385 & $92.5 \%$ & 588 & 1,472 & 6,607 \\
\hline WaPoN & & 33,801 & 566 & 89 & $15.7 x$ & 616 & 0 & 5,243 \\
\hline WaPS (WSU LIBRARY) & & 44,866 & 1,630 & 197 & $12.0 z$ & 2,013 & 1,674 & 19,013 \\
\hline
\end{tabular}

Fig. 2. Washington Library Network Monthly Activity Report (selective sample).

average monthly wage. This average was then divided by 174 (the standard figure for university staff hours per month) to determine the average hourly rate. To calculate staff costs per minute, it was necessary to carry the per-minute costs to the third decimal to approximate the total dollars expended for staffing (see table 7). No other indirect costs, e.g., breaks, annual leave, or holidays, were included in staff wages; however, in order to determine the staff hours available to perform the functions being analyzed, nonproductive hours or staff hours devoted to other assignments had to be calculated and deducted. These calculations were made according to the following formula:

$$
\begin{aligned}
& \text { hours/year } \text { committee assignment (varied) } \\
& \text { hours/year } \text { unit meetings (varied) } \\
& 120 \text { hours/year } \text { breaks (standard) } \\
& \text { hours/year } \text { annual leave (varied) } \\
& 88 \text { hours/year } \text { holidays (standard) } \\
& 96 \text { hours/year } \text { sick leave (standardized) } \\
& \text { based on hours earned per month } \\
& \text { hours/year } \div 12=\ldots \text { hours/month }
\end{aligned}
$$

The primary reasons for variation in the nonproductive hours were length of service and whether a staff member was faculty or classified. Staff costs under the manual system were based on current monthly wages; however, the number and level of staff are essentially that which existed at the time the manual system was functioning (see table 8). Timeslip costs were not based on the minimum hourly wage, since a large number of hours were work/study during the period of the analysis. The total hours worked were divided by the total monthly expenditure to derive the per-minute timeslip costs. No effort was made to reconstruct actual timeslip costs under the manual system, but the same per-minute timeslip costs were used in order to avoid unnecessary skewing of staff costs under the manual system.

\section{DATA BASE COSTS}

The per unit costs of using the WLN bibliographic system, both for performing processes and securing products, were based on the 1979-80 
Table 5. Type and Average Number of Items Searched/Cataloged Per Month on Automated System (Based on WLN Invoice Data and CBP Work Assignment/Staffing Profile)

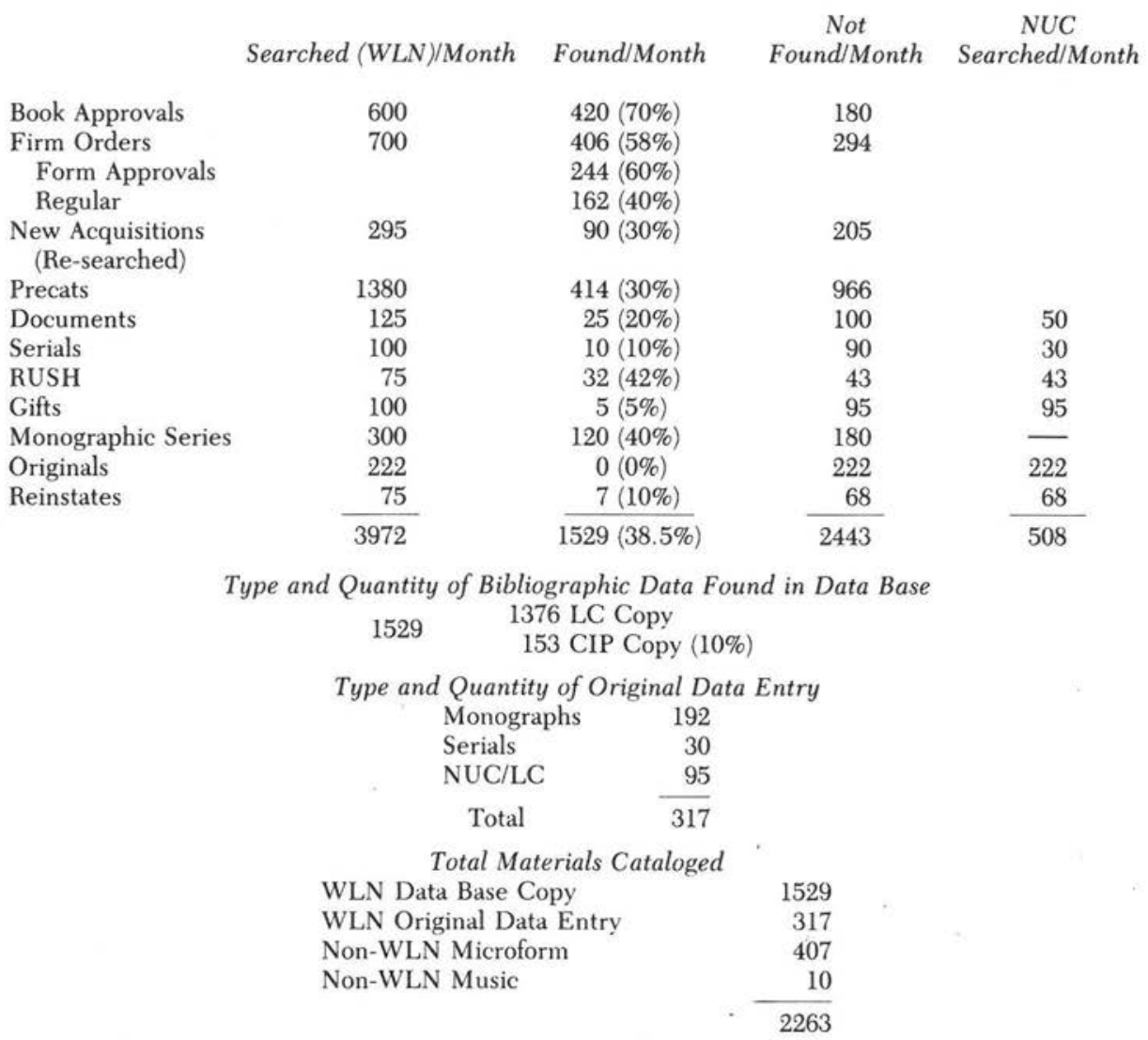

WLN schedule of charges. The average number of items processed was derived from the WLN invoices. The per-record cost of the COM catalog was calculated by taking the total costs of producing the COM cata$\log$ from July 1979 to February 1980 and dividing these costs by the number of titles contained in the COM catalog. Although the WLN schedule of charges stipulates a charge of .069 cents per data-base inquiry, three kinds of processes allow a given number of inquiries without charge. Since not all allowable inquiries are always used for these processes, there are generally a number of inquiries which can be made without charges being assessed. Between July 1979 and February 1980, the average number of monthly inquiries for which there was a charge was 11,800 ; the average number per month for which there was no charge assessed was 8,044. For this reason, in the cost analysis of the automated system (table 2(a)), there appears a category "Items Searched, No Inquiry Charges" under the Bibliographic Searching section. 
Table 6. Type and Average Number of Items Searched/Cataloged Per Month on Manual System (Based on CBP Work Assignment/Staffing Profile)

$$
\begin{array}{llcc} 
& & \text { Not } & \text { NUC } \\
\text { Searched (IDC)/Month } & \text { Found/Month } & \text { Found/Month } & \text { Searched/Month }
\end{array}
$$

$\begin{array}{lrrrr}\text { Book Approvals } & 600 & 300(50 \%) & 300 & \\ \text { Firm Orders } & 700 & 280(40 \%) & 420 & 420 \\ \text { New Acquisitions } & 295 & 59(20 \%) & 236 & \\ \quad \text { (Re-searched) } & & & & \\ \text { Precats } & 1380 & 276(20 \%) & 1104 & 113 \\ \text { Documents } & 125 & 12(10 \%) & 113 & 95 \\ \text { Serials } & 100 & 5(5 \%) & 95 & 52 \\ \text { RUSH } & 75 & 23(30 \%) & 52 & 95 \\ \text { Gifts } & 100 & 5(5 \%) & 95 & 210 \\ \text { Monographic Series } & 300 & 90(30 \%) & 210 & 68 \\ \text { Originals } & 222 & 0(0 \%) & 222 & 1275 \\ \text { Reinstates } & 75 & 7(10 \%) & 68 & 2915 \\ \quad \text { Total } & 3972 & 1057(26.5 \%) & 225\end{array}$

\begin{tabular}{cc} 
Type and Quantity of Materials & Cataloged \\
IDC Copy & 1057 \\
Modified Copy & 984 \\
Original Cataloging & 222 \\
\cline { 2 - 2 } & 2263
\end{tabular}

(Note: Part of the "no charge" inquiries are generated and used by the Acquisitions Unit and are therefore not included in this analysis.)

Although the terminal service and line charges might simply have been added as a total amount to the data-base costs, it seemed more meaningful to distribute these costs on a per-use basis. The method used to distribute these charges was to identify each use of the bibliographic data base, and to divide the total monthly costs of terminals and lines by the total monthly units of use (see table 9). This method of distributing terminal service and line charges not only provided per-unit terminal use costs, but also served to categorize kinds and quantity of data-base use.

\section{SUBSCRIPTION AND MATERIAL COSTS}

Subscription costs include only those bibliographic tools purchased for use in TSD for the purpose of bibliographic searching. As a result of the increased growth of the bibliographic data base, fewer tools are being used for searching under the automated system than under the manual system. Prior to the implementation of WLN, the library subscribed to bibliographic data (LC and CIP copy) on microfiche supplied by the Information Dynamics Corporation (IDC). The per-unit costs of all subscriptions are presented in the cost analysis charts (tables 1 (a) and 2(a)).

Material costs include only those materials unique to cataloging and book processing; general supplies, such as pencils and paper, are not included. The calculation of the per-unit cost of most materials is generally straightforward. It should be noted, however, that under the automated system, products, i.e., materials, are included in the data-base 
Table 7. Staff Costs: Automated Cataloging and Book Processing System

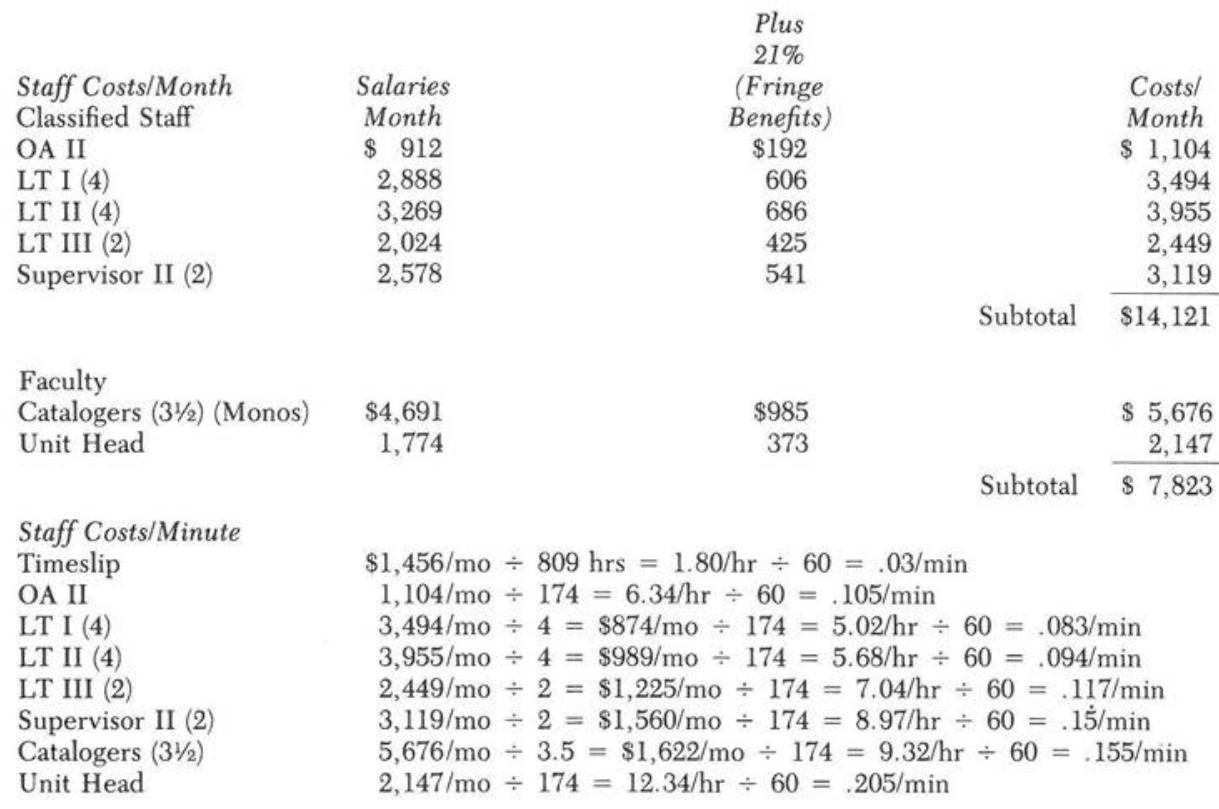

Total Staff Costs/month

Timeslip- $809 \mathrm{hrs}$ @ \$1,456/mo

Special Projects Librarian

Classified Staff

$\$ 1,456$

$345^{*}$

14,121

Faculty

7,823

Total (All Staff)

$\$ 23,745$

*Amount of time (wages) assigned to cataloging.

costs, and only those materials used independent of the data base, e.g., book pockets and book cards, are listed as material costs on the charts. Under the manual system, due to the divisional arrangement of the library system and the number of card catalogs being maintained, the formula for producing sets of cards for a single title was complex. For this reason, the costs and number of cards produced for the titles cataloged per month are listed as a separate line item.

\section{EQUIPMENT COSTS}

Equipment costs include only equipment unique to cataloging and book processing, i.e., required for processing or products. General equipment, such as desks, book trucks, typewriters, are not included.

\section{Equipment-Automated System}

During the period covered by the cost analysis, November 1977 to February 1980, the following equipment was purchased for the automated system: 
7 Bibliographic terminals

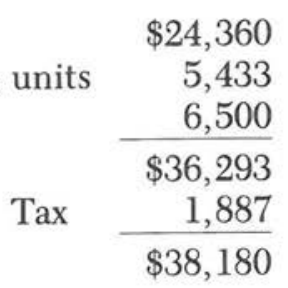

Two pieces of equipment are currently being leased (maintenance included):

$$
\begin{array}{ll}
\text { Keypunch } & @ \$ 92.61 \\
\text { Verifier } & @ 101.12 \\
& \$ 193.73 / \text { month }
\end{array}
$$

\section{Summary of Monthly Equipment Costs}

Purchases (5-year amortization) $\$ 636.33$

Maintenance $\quad 60.00$

Leased equipment

$$
193.73
$$

$\$ 890.06 /$ month

\section{Equipment-Manual System}

If the automated system had not been implemented, the following equipment would have been purchased during this period:

$$
\begin{array}{lr}
2 \text { Card catalogs } & \$ 3,755 \\
5 \text { Kardex units } & 4,475 \\
2 \text { Linedex units } & 2,944 \\
\cline { 2 - 2 } \text { Tax } & \$ 11,174 \\
\cline { 2 - 2 } & \$ 11,755
\end{array}
$$

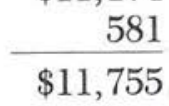

Although the anticipated life span of this equipment should be considerably greater than that of terminals and modems, it has also been amortized over a five-year period. The rationale for this period of amortization is that the rate of growth of the files for which the equipment is used results in the purchase of additional equipment equivalent to the expected replacement of electronic equipment. Therefore, the initial cost of these purchases amortized would have been $\$ 196 /$ month.

Since the multilith has been owned by the library for more than twenty years, its purchase price is not applicable to this analysis. However, maintenance on the multilith is $\$ 72.24 /$ month. Two pieces of equipment were being leased under the manual system (maintenance included):

$$
\begin{array}{ll}
\text { Keypunch } \\
\text { Verifier }
\end{array} \frac{101.12}{\$ 193.73 / \text { month }}
$$




\section{Summary of Monthly Equipment Costs}

Purchases (5-year amortization) $\$ 196.00$

Maintenance

72.27

Leased equipment

193.73

$\$ 462.00 /$ month

\section{SUMMARY AND CONCLUSION}

The cost analysis clearly indicates that at Washington State University Libraries the automated cataloging and book processing system is less expensive than its previous manual system. By using the bibliographic component of the Washington Library Network, the library has reduced the costs of searching, cataloging, and record maintenance by almost 20 percent (see table 10-summary comparison costs by function). The higher costs of the manual system are essentially staff costs. Under that

Table 8. Staff Costs: Manual Cataloging and Book Processing System (Based on the 1977 Staffing Levels at Current Staff Costs)

$\begin{array}{lc} & \\ \text { Staff Costs/Month } & \text { Salaries } \\ \text { Classified Staff } & \text { Month } \\ \text { OA II-Typing } & \$ 912 \\ \text { LT I (11) } & 7,950 \\ \text { LT II (3) } & 2,434 \\ \text { LT III (5) } & 5,060 \\ \text { Supervisor I (2) } & 2,175 \\ \text { Supervisor II } & 1,289 \\ \text { Offset Duplicator Operator } & 1,135\end{array}$

Plus

$21 \%$

(Fringe

Benefits)

\& 192

1,670 .

Costs/

912

7,950

511

S 1,104

1,063

9,622

5,060

2,175

457

2,945

1,289

271

238

6,123

2,632

1,560

1,373

Subtotal

$\$ 25,359$

Faculty

Catalogers (3.5)

4,691

1,774

985

373

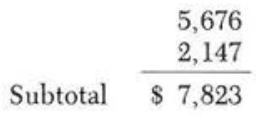

Staff Costs/Minute

Timeslip

OA II-Typing

LT I (11)

LT II (3)

LT III (5)

Supervisor I (2)

Supervisor II

Offset Duplicator Operator

Catalogers (3.5)

Unit Head

$\$ 2,174 / \mathrm{mo} \div 1208 \mathrm{hrs} .=1.80 / \mathrm{hr} \div 60=.03 / \mathrm{min}$.

$1,104 / \mathrm{mo} \div 174=6.34 / \mathrm{hr} \div 60=.105 / \mathrm{min}$

$9,622 / \mathrm{mo} \div 11=875 / \mathrm{mo} \div 174=5.03 / \mathrm{hr} \div 60=.084 / \mathrm{min}$

$2,945 / \mathrm{mo} \div 3=982 / \mathrm{mo} \div 174=5.64 / \mathrm{hr} \div 60=.094 / \mathrm{min}$

$6,123 / \mathrm{mo} \div 5=1,225 / \mathrm{mo} \div 174=7.04 / \mathrm{hr} \div 60=.117 / \mathrm{min}$

$2,632 / \mathrm{mo} \div 2=1,316 / \mathrm{mo} \div 174=7.56 / \mathrm{hr} \div 60=.126 / \mathrm{min}$

$1,560 / \mathrm{mo} \div 174=8.97 / \mathrm{hr} \div 60=.149 / \mathrm{min}$.

$1,373 / \mathrm{mo} \div 174=7.89 / \mathrm{hr} \div 60=.13 / \mathrm{min}$

$5,846 / \mathrm{mo} \div 3.5=1,670 / \mathrm{mo} \div 174=9.60 / \mathrm{hr} \div 60=.155 / \mathrm{min}$

$2,147 / \mathrm{mo} \div 174=12.34 / \mathrm{hr} \div 60=.205 / \mathrm{min}$.

Total Staff Costs/Month

Timeslip- $1208 \mathrm{hrs}$

@ $\$ 2,174 / \mathrm{mo} \$ 2,174$

Classified Staff

Faculty

7,823
35,356

Total (All Staff) 
Table 9. Bibliographic Data Base Use Per Month (One Unit $=$ One Access to or Process in Data Base)

\section{Category}

Searching

Cataloging (Data Base Copy)

Cataloging (Original Data Entry)

Authority Verification $(317 \times 7)$

Bibliographic Changes/Corrections

ILL, REF, General
Quantity of Terminal Use

\begin{tabular}{cr} 
& 10688 \\
& 1529 \\
& 317 \\
& 2219 \\
& 360 \\
& 537 \\
\cline { 2 - 2 } Total Units $\quad 15650$
\end{tabular}

WLN Terminal Service and Telecommunication Line Charges/Month \begin{tabular}{ll}
$51 / 2$ terminals@ $\$ 140 / \mathrm{mo}$ & $=\$ 770 / \mathrm{mo}$ \\
$51 / 2$ lines@ $\$ 40 / \mathrm{mo}$ & $=220 / \mathrm{mo}$ \\
\cline { 2 - 3 } & $\$ 990 / \mathrm{mo}$
\end{tabular} $\$ 990 \div 15650=\$ .06 /$ terminal use for Cataloging and Book Processing System

Table 10. Cataloging and Book Processing System: Summary Comparison Costs by Function (Excluding Equipment Costs)

Function

$\begin{array}{cc}\text { Number of } & \text { Costs Per } \\ \text { Items } & \text { Month }\end{array}$

Manual System

1. Bibliographic Searching

2. Bibliographic Record Production (Cost of Catalog Cards Distribution)

$[2263]^{*}$

LC copy cataloging

1057

Modified copy cataloging

Original cataloging

222

Miscellaneous

3. Bibliographic Record Maintenance

4. Marking

Total

\begin{tabular}{c}
$\$ 4,617$ \\
{$[\$ 13,143] \dagger$} \\
4,092 \\
5,021 \\
3,343 \\
687 \\
6,402 \\
4,631 \\
\hline$\$ 28,793$
\end{tabular}

Automated System

1. Bibliographic Searching

2. Bibliographic Record Production (Cost of Catalog Cards Included)

LC and CIP copy cataloging

Modified copy cataloging

NA

NA

NA

Original cataloging

Miscellaneous

3. Bibliographic Record Maintenance

4. Marking

Total

\begin{tabular}{|c|c|}
\hline 4480 & $\$ 2,170$ \\
\hline [2263]* & {$[\$ 11,837] \dagger$} \\
\hline 1529 & 4,900 \\
\hline 512 & 1,523 \\
\hline 222 & 3,343 \\
\hline NA & 2,071 \\
\hline NA & 4,194 \\
\hline $\mathrm{NA}$ & 4,589 \\
\hline
\end{tabular}

*Total of items listed below.

$†$ Total of costs listed below.

system, eleven more staff and 1,365 more timeslip hours were needed per month to process the same amount of materials as is processed under the automated system. In fact, compared to the staff costs of both the manual and automated systems, the costs of equipment, data-base use (including products), terminal service, and telecommunication lines 
of the automated system are a relatively small percentage ( 27 percent) of the total cataloging and book processing costs. This analysis serves to underscore a basic reality of the current library organization: personnel is one of its largest expenditures and staff-intensive systems are very costly.

This cost analysis has not directly addressed the issue of the quality of processing and products of either the manual or automated systems. The analysis suggests, however, that the automated system is more efficient in terms of staff time. Moreover, the TSD staff has found that not only can more be done with fewer staff, but the automated system also provides more accurate data and has the flexibility to accommodate with relative ease the many corrections and changes that must be made to the library's bibliographic files.

Joselyn Druschel is assistant director for automation and technical support at the Washington State University Libraries. She is currently chairing a staff task force which is developing specifications for the Libraries' on-line catalog. 